\title{
Performing Active Noise Control and Acoustic Experiments Remotely
}

\author{
http://dx.doi.org/10.3991/ijoe.v8iS4.2304 \\ I. Khan, D. Muthusamy, W. Ahmad, B. Sällberg, K. Nilsson, J. Zackrisson,I. Gustavsson and L. Håkansson \\ Blekinge Institute of Technology, Karlskrona, Sweden
}

\begin{abstract}
This paper presents a novel and advanced remotely controlled laboratory for conducting Active Noise Control (ANC), acoustic and Digital Signal Processing (DSP) experiments. The laboratory facility, recently developed by Blekinge Institute of Technology (BTH) Sweden, supports remote learning through internet covering beginners level such as simple experimental measurements to advanced users and even researchers such as algorithm development and their performance evaluation on DSP. The required software development for ANC algorithms and equipment control are carried out anywhere in the world remotely from an internet-connected client PC using a standard web browser. The paper describes in detail how ANC, acoustic and DSP experiments can be performed remotely The necessary steps involved in an ANC experiment such as validity of ANC, forward path estimation and active control applied to a broad band random noise [0$200 \mathrm{~Hz}]$ in a ventilation duct will be described in detail. The limitations and challenges such as the forward path and nonlinearities pertinent to the remote laboratory setup will be described for the guidance of the user. Based on the acoustic properties of the ventilation duct some of the possible acoustic experiments such as mode shapes analysis and standing waves analysis etc. will also be discussed in the paper.
\end{abstract}

Index Terms-Active Noise Control, LMS, Remote Laboratories, VISIR.

\section{INTRODUCTION}

Students require a considerable amount of hands-on experience in Engineering Education in general and particularly in the fields of Active Noise Control (ANC) and acoustics. Hands-on experience is needed both for an in-depth knowledge and future employment benefits. The experimental setup required for ANC experiments broadly consists of an application test bed or device under test (DUT), Digital Signal Processor (DSP) board along with transducers, data acquisition and analysis equipment supported by computer based analysis tools. These equipment are expensive and usually a single set is available to the students in a time limited scheduled laboratory session. With the increasing number of students and limited financial resources many universities around the world have complemented some of their traditional laboratories with remotely controlled laboratories.

This article presents a novel and advanced laboratory system for conducting ANC, acoustic and DSP experiments remotely over the internet. Although the proposed system is primarily focused on active noise control in a ventilation duct, the system also allows general experiments in the field of acoustics and digital signal processor.
The proposed laboratory concept is based on standard low cost components and interfaces and brings new knowledge and benefits to the community of remote laboratories

\section{A. Remotely Controlled Laboratories}

Educational laboratories can be divided into three categories namely as traditional hands-on laboratories with real equipment and system, simulation based laboratories and remotely controlled laboratories. The term remotely controlled, online, distance or web laboratories refer to the class of educational laboratory setups where the actual equipment and systems are accessed and controlled remotely through the internet [1]. The interaction with the systems and equipment is provided through a client interface e.g. in a web browser, remote controlled switches and virtual instrumentation tools while remote monitoring is provided by audio/video support [2].

\section{B. Traditional, Simulation Based or Remotely Controled Laboratories}

There is a general consensus on the important role of laboratory education in engineering but a clear disagreement on the suitability of each kind of laboratory [1][2]. This discussion has failed to reach any solid conclusion as each laboratory has its own qualities, capacity, restrictions and also its own advocators and detractors. Accreditation boards, engineering professional ethics and current industrial development require physical interaction with real world systems and equipment making traditional hands-on laboratories more favorable [3]. Simulations and modeling based laboratories which are mostly suitable for development and design fail to fulfill the aforementioned requirements. Traditional hands-on laboratories are expensive and not sufficient for the ever-increasing number of students and decreasing budget. A remote laboratory is an attractive alternative as it provide $24 / 7$ access. The initial costs as well as the operational costs are reduced significantly, as only a single setup is installed with reduced maintenance and supervision costs without compromising on the objectives of laboratory education as mentioned in [3]. It is worthwhile to mention that most of the remote laboratories are based on individual efforts. This created compatibility and reusability issues due to lack of standards and common communication protocols. These issues along with others such as comparison, advantages, educational effectiveness and limitations of all the three types of laboratories are discussed thoroughly in $[2][3][4][5][6][7][8][9]$.

\section{A Brief History of Remotely Controlled Laboratories}

Digitalization of laboratory equipment and the use of computers can be seen in laboratory education since 1972 
and mid 1980s [3]. With the arrival of World Wide Web and internet, online education has taken over the once widely used term "distance education". Laboratories also came online with the emergence of computerized remotely controlled data acquisition, measurement and analysis systems. There have been numerous individual as well as collaborative efforts by universities across the globe to develop remote laboratories based on different architectures and software tools [2][6][10].

\section{The VISIR Project by BTH}

Interest in remote laboratories at Blekinge Institute of Technology (BTH) Sweden had started in the form of a feasibility study in 1999 followed by the Virtual Instruments System in Reality (VISIR) project in 2006 [9]. The VISIR project aims to develop remote laboratories cooperating as nodes in a grid on the internet, based on open source software platforms and standard Instrumentation platforms for the measurement and analysis equipment. The idea attracted several universities from Europe and Asia to cooperate and develop remote laboratories [5][11][12]. A number of remote laboratories have been developed by BTH and partner universities in the fields of electronic circuits, vibration analysis, antenna theory, telecommunication, computer sciences etc. The laboratory system presented in this paper is based on the success of the VISIR project and thus highly flexible.

\section{THE REMOtEly CONTROLLED ANC LABORATORY SETUP AT BTH}

The remote ANC laboratory is based on the VISIR open source platform [9], National Instruments (NI) LabVIEW software and a DSP board TMS320C6713DSK from Texas Instruments (TI). The test bed or DUT for active control consists of a ventilation duct along with speakers and microphones for ventilation noise control. The laboratory setup is divided into two main categories, i.e., the actual hardware or equipment in the laboratory, and the graphical user interface presented remotely to the user on his/her computer screen for configuration and control of these equipment.

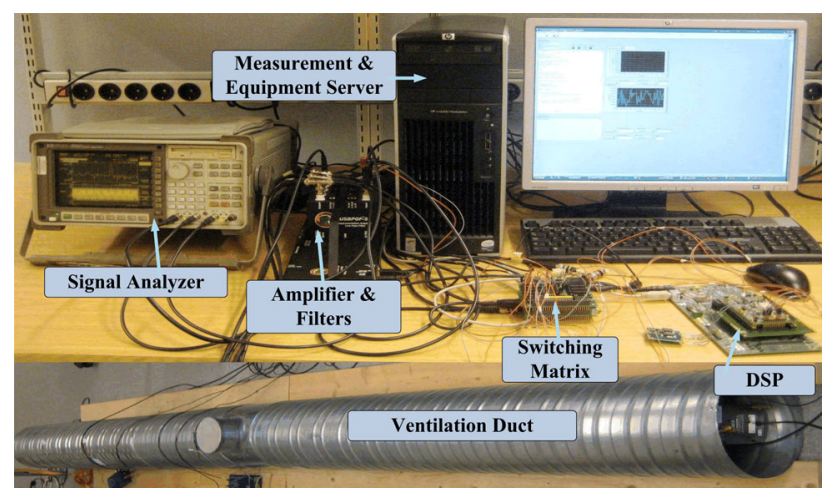

Figure 1. A snap shot of the equipment present in the remote ANC laboratory

\section{A. The Actual Equipment in the Laboratory}

Fig. 1, shows the hardware and equipment present in the proposed ANC laboratory. The equipment is classified into three groups based on their functionality as explained in the subsequent sections. The interconnection between the equipment and a user through the internet is shown in Fig. 2.

\section{1) Active Noise Control, Analysis and DSP}

The initial laboratory setup is aimed at a single channel feed forward ANC applied to a ventilation duct, as discussed in [13]. A $4.05 \mathrm{~m}$ long circular ventilation duct with an inner diameter of $315 \mathrm{~mm}$ is used. Inside the duct are four reference microphones and one error microphone of ordinary quality (VM-6052-5382) fixed. Two loudspeakers (Fostex-6301B3) are placed at each end of the ventilation duct, one acting as source (primary noise) and the other as control (secondary noise) speaker during ANC. A four channel dynamic signal analyzer (HP36570A) is used for analysis of the control and measurement signals as well as acting signal source to the primary noise speaker. The adaptive control algorithms are implemented on a TMS320C6713DSK from Texas Instruments. The filter/amplifier module USBPGF-S1/L from Alligator Technologies is used for the signal conditioning of the signals. A daughter card module (S. Module 16-100) from

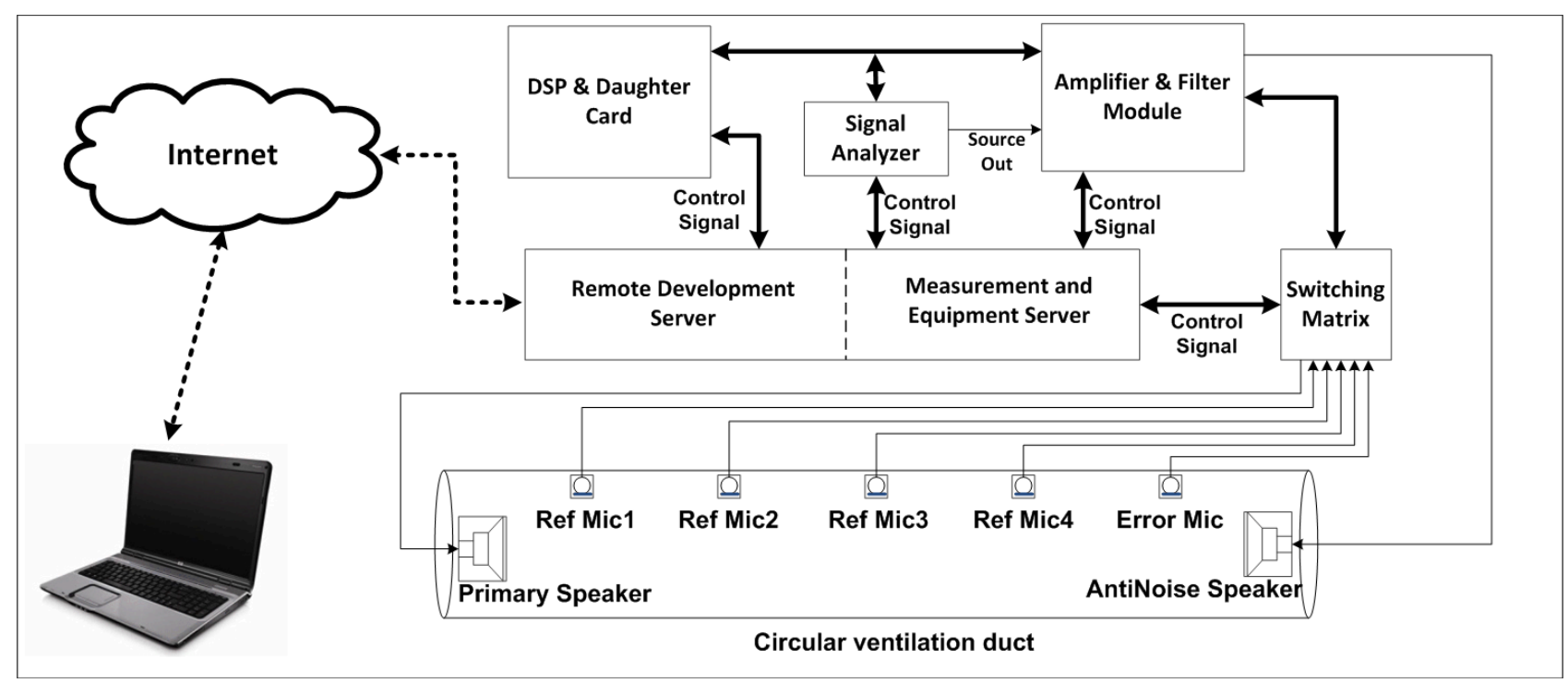

Figure 2. Block diagram of the remote ANC laboratory showing the interconnection of different equipment to the servers and to the user via internet. 
SEMATIC connected to the DSP board through a minibus interface is responsible for low-latency 16 bit analog to digital and digital to analog conversion. The remote configuration of the equipment, as required by a particular ANC and acoustic experiment, is achieved by a relay switching matrix also referred to as a Telemanipulator, used in other remote laboratories developed under VISIR[13].

\section{2) Measurement and Equipment Server}

The remote ANC laboratory is based on a client-server architecture, inherited from its predecessors developed under the VISIR. The measurement and equipment server is an HP Workstation with Windows XP acts as a link between the laboratory equipment and the internet, as shown in Fig. 2. It also acts as web server and hosts the client interface discussed in the following section. The signal analyzer is connected to the measurement and equipment server through a General Purpose Interface Bus (GPIB) while the switching matrix and the Filter/Amplifier module USBPGF-S1/L is accessible via a Universal Serial Bus (USB) interface. Finally the DSP is connected to the server via the Joint Test Action Group (JTAG) interface.

\section{B. Remote Graphical User Interface}

A graphical user interface is an essential part of a remote laboratory since it provides the necessary means of interaction between the student and the hardware placed in the laboratory. Efforts are made to make this interaction appear as genuine as possible to convince the student that he/she is working with real equipment and not simulations. The ANC laboratory provides two such interfaces named as the Measurement and Configuration Client and the Web-Based Development Environment. When the laboratory is accessed only the Measurement and Configuration Client is presented to the user and the WebBased Development Environment is launched from Measurement and Configuration Client when needed.

\section{1) Measurement and Configuration Client}

The Measurement and Configuration Client, as shown in Fig. 3, presents the ANC laboratory equipment to the student in the client's web browser. The client interface is

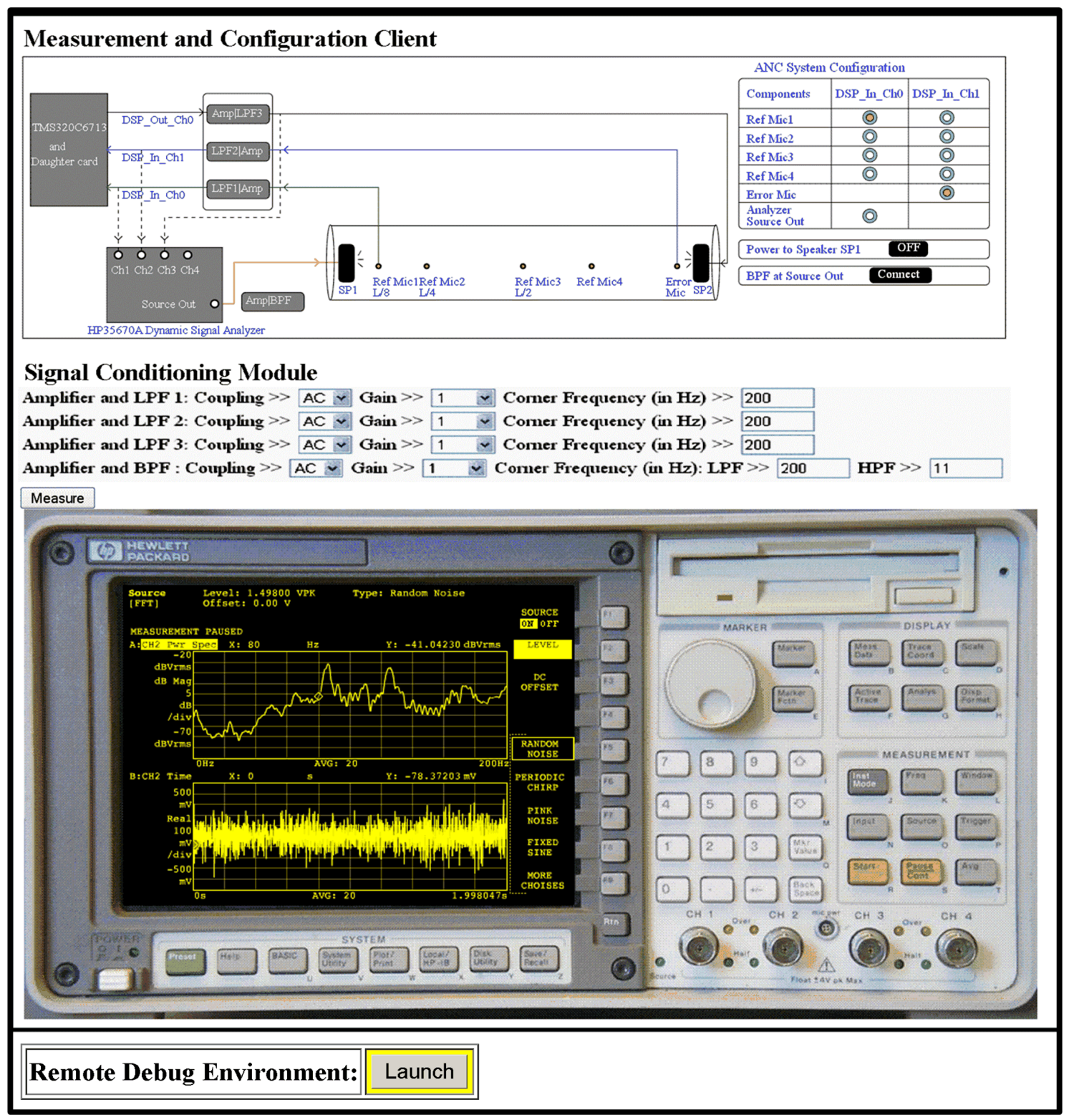

Figure 3. The Measurement and configuration Client shown at the user computer in a standard web browser. 
developed using Hyper Text Mark Language (HTML), Java script and Adobe Flash. Three distinct areas are easily identified in the client interface. The top right part, i.e., the ANC System Configuration tab is used to select the desired microphones, loudspeakers in the duct and connect them to a DSP channel according to the requirements of experiment at hand.

Clicking the appropriate "Radio button" connects the desired microphone to the corresponding DSP channel through the filter/amplifier module and the data acquisition card. The central part of the client denoted as Signal Conditioning Module provides access to the filter/amplifier module. The necessary amplification and filtering (anti-aliasing and re-construction) of the signals from the microphones and control signals from the DSP are performed in this section. Other parameters such as AC/DC coupling, amplifier gain and corner frequency for the anti-aliasing and re-construction filters are easily selected from the corresponding "drop down" menus. The bottom part of the client is a front panel of the signal analyzer HP36570A, developed in Adobe Flash at BTH. The Adobe Flash front panel provides almost all of the functionalities of the four channel dynamic Signal Analyzer the user may need for the analysis and measurement of the signals during ANC and acoustic experiments.

\section{2) Web-Based Development Environment}

The Web-Based Development Environment is a Remote Development Environment (RDE) which steers the DSP board from a client computer over the internet. This remote access feature is not present in the DSP provider's Integrated Development Environment (IDE) the Code Composer Studio (CCS). The RDE provides a user the core functionalities of CCS such as file operations project management, compiling the source code, downloading the executable to the DSP and a series of debugging features remotely. The integrated core functionalities of CCS in the RDE are made remotely available using LabVIEW's. DSP Test Integration tool kit and Web publishing tool [14]. In the back ground the web-based development environment uses the CCS IDE on the server to enable the student to develop and debug any program to be downloaded and executed on the DSP. The RDE is launched by clicking the "Launch" button at the bottom of the Measurement and Configuration Client. The RDE also provides the functionality to plot memory variables during code execution on the DSP and users can read and update the variables in the algorithm while performing the experiment. A snapshot of RDE is shown in Fig. 4.

\section{ACtive Noise Control ApPliEd to A VENTILATION DUCT}

Active noise control applied to ventilation duct is a classical and well understood example of ANC and may be used to illustrate the concepts and challenges of ANC in field of active control [15]. Although the ANC laboratory is a multifunctional platform, the main focus is on ANC experiments applied to a ventilation duct. In this section some basic ANC concepts followed by the necessary steps in an ANC experiment on the remote laboratory are discussed.

\section{A. Active Noise Control}

Noise attenuation in ventilation ducts by passive silencers is generally not practical for low frequency noise. To provide adequate attenuation in the low-frequency range, passive silencers at these frequencies tends to be large and bulky as the acoustic wavelengths are long in this frequency range [16][17]. ANC methods provide good attenuation at low frequencies in the plane wave region. The main goal of ANC is to attenuate low frequency noise known as primary noise, by producing an anti-noise (sound) generated by a loudspeaker referred to as secondary sound source. The idea was first conceived by Paul Leug in 1936 in a United States patent, which failed to materialize but later on received considerable attention from researchers in the late 1970's [15]. ANC methods can be further classified as feed forward, feedback or hybrid based on the control strategy employed [15][16]. The presented remote ANC laboratory focuses on a single channel feed forward ANC method applied to low frequency noise in ventilation duct. The single channel approach implies the use of a single loudspeaker as the secondary sound source while feed forward means that the controller which may be an FIR or IIR filter, is supplied with some prior information of the noise, known as a reference signal. The signal analyzer is used to generate the primary noise for the duct which can be conditioned if desired while the secondary loudspeaker is steered by the controller. For the case of e.g. control of non-stationary random noise, the controller need to be adaptive to the changes and usually an adaptive algorithm based on the Least Mean Square (LMS) algorithm is employed in the controller to keep track of non-stationary behavior of the primary noise [15][18][19].

Fig. 5, shows a simplified schematic diagram of a single channel feed forward ANC applied to the ventilation duct. The reference signal $x(t)$, sensed by the reference microphone and error signal $e(t)$ from the error microphone are fed to the adaptive controller denoted as ANC. The output of the controller $y(t)$, in time domain controls the secondary loudspeaker via an amplifier and is the secondary noise or control signal. Fig. 6, shows the detailed version of the same ANC applied to the ventilation duct in the remote ANC laboratory. It is obvious from Fig. 6, that there are some physical components between the control signal $y(n)$ from the controller (in the digital domain) and the error signal $e(t)$ from the error microphone. The presence of these components introduce a dynamic system also known as forward path which need to be controlled by the adaptive controller. The signals are converted to digital or time domain by $\mathrm{A} / \mathrm{D}$ or $\mathrm{D} / \mathrm{A}$ converters and reconstruction filters as required by a particular component. The sampled digital signals available and produced by the DSP are denoted, for simplicity, as $x(n)$, $d(n), y(n)$ and $e(n)$, respectively, where $n$ refers to the discrete time sample index. If the sampling frequency is denoted by $F_{z}$ in $H z$, then the sample time points $\mathbf{t}_{\mathbf{n}}$ in seconds are given by thus $t_{n}=\frac{n}{F_{s}}$.

The LMS algorithm is not defined for applications where its output signal is used as a control signal or input signal to a dynamic system where the output signal of the dynamic system forms an estimate of a desired signal, thus in ANC applications a modified version of the LMS algorithm i.e. the filtered-x LMS algorithm is frequently used [15]. A block diagram illustrating the Filtered-x LMS 


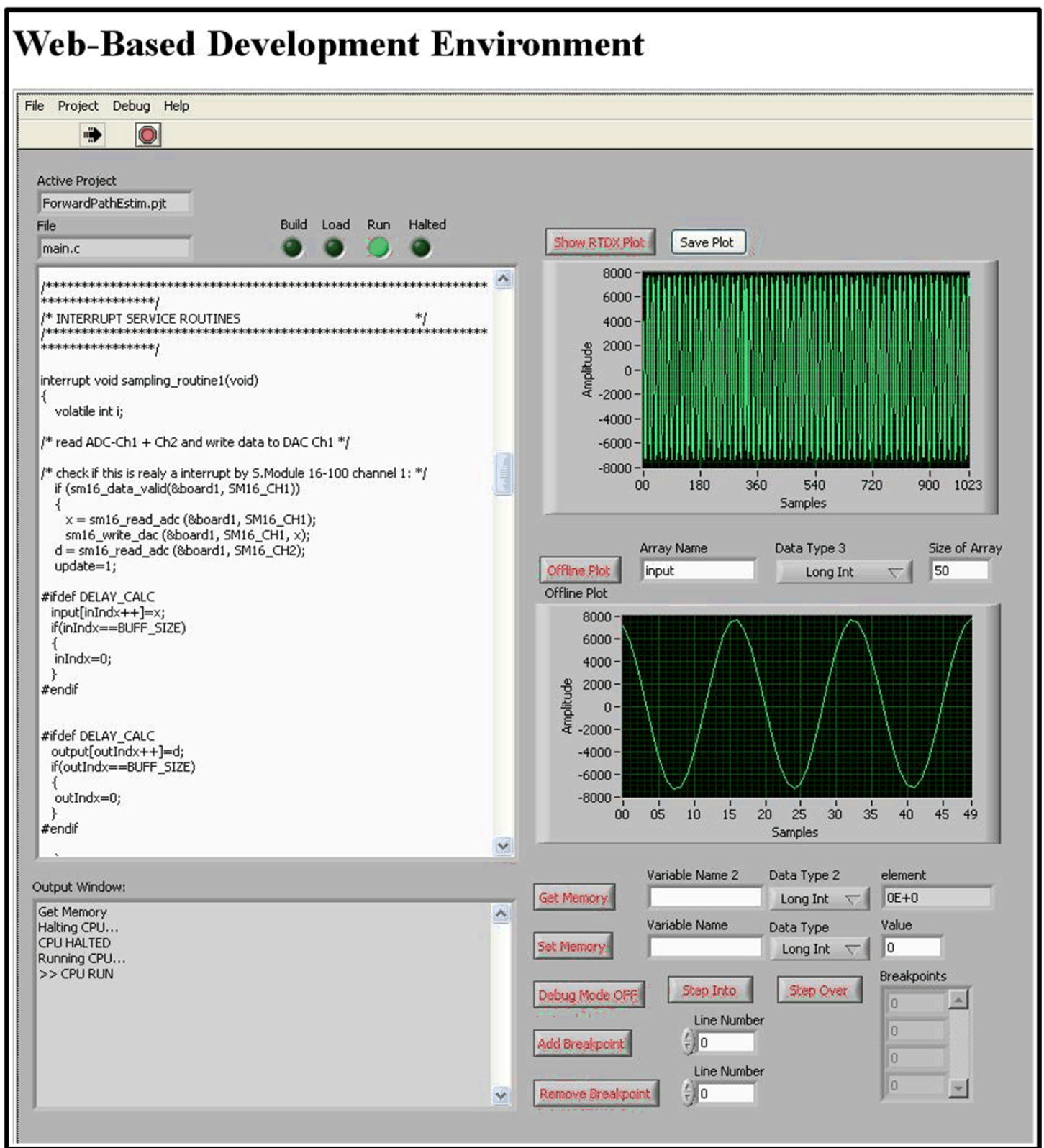

Figure 4. Screen shot of the Remote Development Environment showing a sample code and other useful functionalities extracted from the extracted from the CCS IDE.

algorithm involving a dynamic system is shown in Fig. 7, and given by the equations [15]

$$
\begin{aligned}
& y(n)=\mathbf{w}_{\mathrm{n}}^{\mathrm{T}} \mathbf{x}(n) \\
& e(n)=d(n)-y_{C}(n) \\
& \mathbf{w}_{n+1}=\mathbf{w}_{n}+2 \mu e(n) \mathbf{x}_{c^{\prime}}(n)
\end{aligned}
$$

Where

$\mathbf{w}(\mathbf{n})=\left[\mathbf{w}_{0}(\mathbf{n}), \quad \mathbf{w}_{1}(\mathbf{n}), \quad \ldots, \quad \mathbf{w}_{\mathrm{M}}(\mathbf{n})\right]$ is the weight vector of the adaptive FIR filter, $\mathbf{x}(\mathbf{n})=[\mathbf{x}(\mathbf{n}), \quad \mathbf{x}(\mathbf{n}-\mathbf{1}), \quad \ldots, \quad \mathbf{x}(\mathbf{n}-\mathbf{M})]$ is the reference signal vector, $y_{c^{\prime}}(n)$ is the output signal of the dynamic system, $d(n)$ is the desired signal or the primary noise to be attenuated by the ANC, $y(n)$ is the output signal of the adaptive FIR filter, $e(n)$ is the error signal and $\mu$ is the step size, $\mathbf{x}_{c^{\prime}}(n)$ is the filtered reference signal vector given by

$$
\mathbf{x}_{c^{\prime}}(n)=\left[\begin{array}{c}
\sum_{l=0}^{L-1} c^{\prime}(l) x(n-l) \\
\sum_{l=0}^{L-1} c^{\prime}(l) x(n-l-1) \\
\cdot \\
\cdot \\
\cdot \\
\sum_{l=0}^{L-1} c^{\prime}(l) \times(n-l-M)
\end{array}\right]
$$

Where $c^{\prime}(l), l=0,1, \ldots . . L$ is a L coefficients FIR filter estimate of the forward path. 


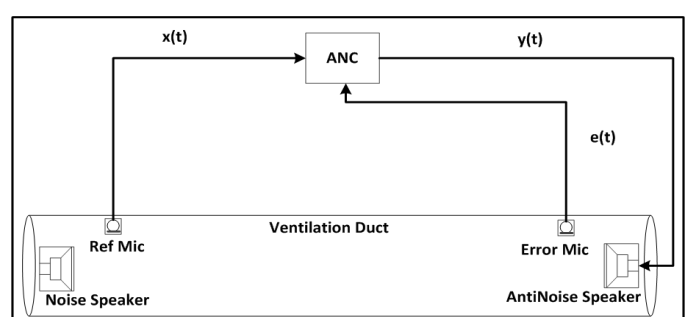

Figure 5. Schematic diagram of a single channel feed forward ANC applied to ventilation duct.

The algorithm adjust the weights with the aim to minimize of the mean square error $E\left[e^{2}(n)\right]$. The weightvector update equation defined in (3) is iteratively used to find filter weights representing the solution to the WienerHopf equations [18].

\section{B. The Forward Path}

Basically in SISO ANC the system to be controlled is the so called forward path, secondary path or control path and this dynamic system may be defined as the system between the controller signal $y(n)$ and the error microphone $e(n)$. The forward path comprises of a number of sub-paths and expanded in terms these paths it may be expressed, D/A converter, reconstruction filter loudspeaker amplifier, secondary loudspeaker, acoustic path between secondary loudspeaker and error microphone, error microphone, error microphone, amplifier, antialiasing filter, and A/D converter. Fig. 8, show the forward path along with the feedback path that is discussed in the subsequent section.

The dynamic system or forward path due to the aforementioned components will filter the control signal $y\left(\mathrm{r}_{\mathrm{l}}\right)$ and introduces frequency dependent phase shift or delay and amplification while forming its output signal $y_{C}(t)$ which is sensed by the error microphone.

\section{System Identification to Estimate the Forward Path}

The forward path may be estimated by an LMS adaptive controller as an FIR filter using a band limited random noise as identification signal prior to ANC implementation. The whole process is known as system identification. A random noise signal $x(t)$ generated by the signal analyzer in the range $[0-200] \mathrm{Hz}$ is used as input signal to the secondary loudspeaker to excite the dynamic system or path that is of interest to identify while the primary speaker is kept off as it is not needed. In Fig. 7, a block diagram illustrates the system for forward path identification. The signal from the error microphone is the desired signal $d(n)$. The LMS algorithm adjusts the adaptive filter coefficients $w_{l}(n), l=0,1, \ldots, \underline{E}$ where $L+1$ is the length of the adaptive FIR filter, as such to minimize the mean square error $E\left[e^{z}(n)\right]$ between the desired signal $d(n)$ and the output of the adaptive filter $y(n)$ and when the adaptive FIR filter has converged it form an FIR filter estimate of the forward path. Fig. 9, shows the configuration of the Measurement and Configuration Client during forward path estimation by the user. After completing the required configuration of the equipment, the RDE is launched to implement the code for the LMS algorithm on the DSP. The student creates a new

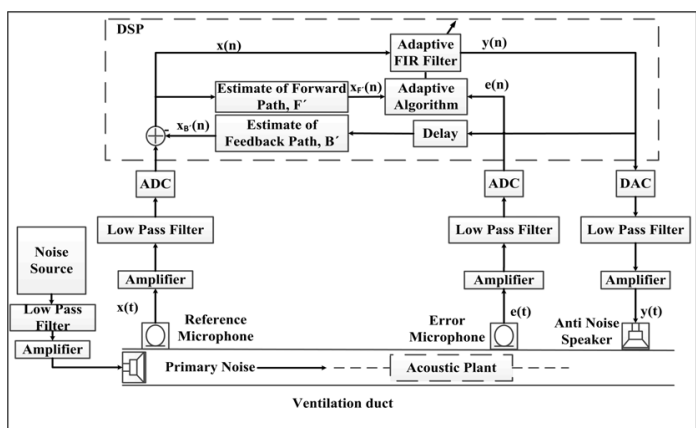

Figure 6. Schematic diagram of the ANC applied to ventilation duct noise showing the equipment involved.

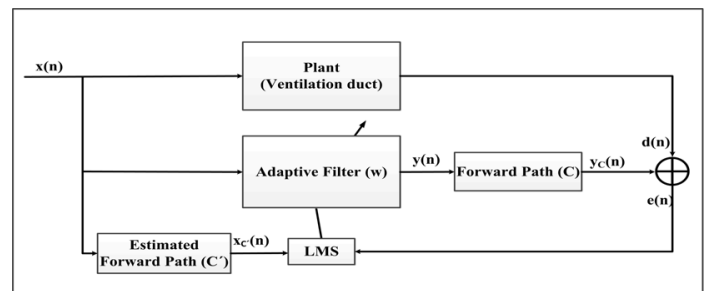

Figure 7. Block diagram of the filtered-x LMS algorithm applied for the control of ventilation noise in a ventilation duct.

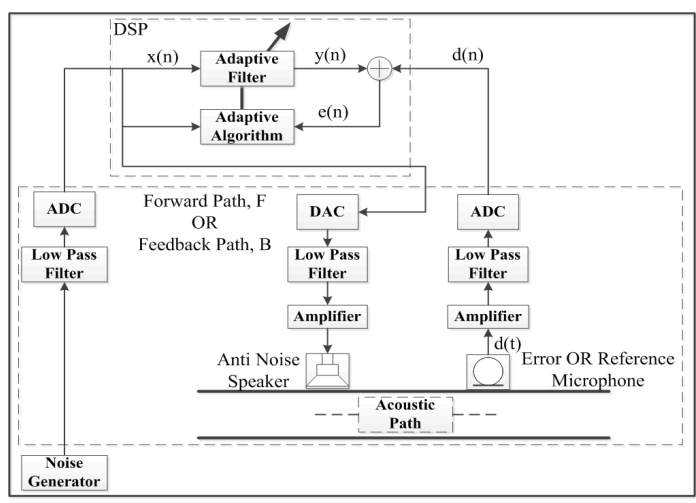

Figure 8. Schematic of the ANC laboratory setup showing the forward path and feedback path.

project and writes his code in the editor area of the RDE. Other functionalities such as Build, Load, Run and Halt are executed form the menus provided. The user code is saved on the server at a dedicated location which can later be retrieved if required. After the adaptive filter has converged, the coefficients of the FIR filter representing the forward path are plotted and saved for later use in the ANC implementation. Similar to the forward path, a feedback path also exist from the output of the controller $y(n)$ to the reference microphone signal $x(n)$. This path include the conditioning equipment between the output of the controller and secondary loud speaker including the D/A converters, secondary loudspeaker, the acoustic path between secondary loudspeaker and reference microphone, the reference microphone and finally the conditioning devices between the reference microphone and the controller including the A/D converters. This feedback path effect can be neutralized by a feedback neutralization filter $B^{*}$ estimated similar to forward path filter. The control signal $y(n)$ is filtered to get the signal $x_{B^{\prime}}(n)$ and it is subtracted from the reference signal $x(n)$ to compensate for the true feedback path in the ANC control system. 


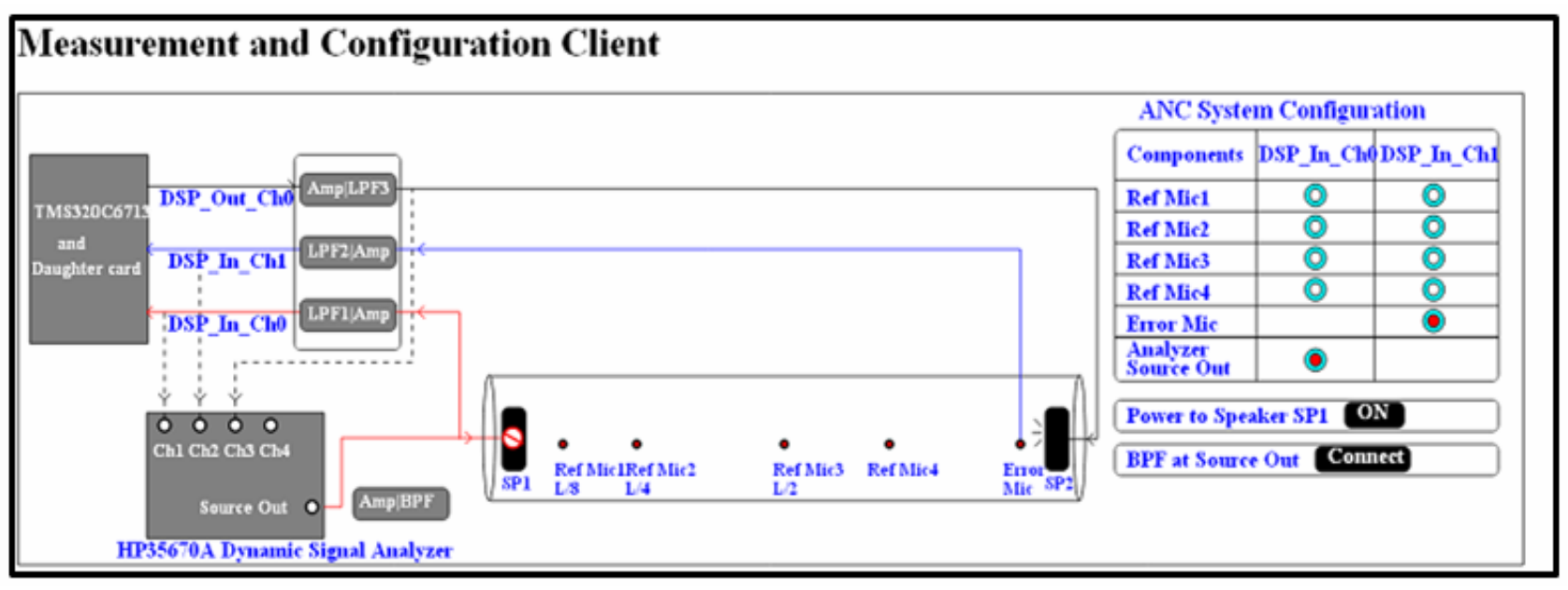

Figure 9. Screen shot of the Measurement and Configuration Client showing the ANC System Configuration tab during forward path estimation.

\section{Feasibility of ANC}

Before applying ANC to any system it is important to know the validity of ANC for the system [16]. The frequency limit for the DUT, i.e., the ventilation duct must be verified for a plane waves propagation so that a single channel ANC can be used.

This frequency limit which is dependent on the dimension of the duct is calculated from the modified wave equation and substituting the boundary conditions for the circular duct [20].

$$
f<\left(\frac{1.84 c}{\pi D}\right)\left(\sqrt{1-\left(\frac{U}{c}\right)^{2}}\right)
$$

Where $f$ is the upper cut-on frequency of the noise in the duct below which there is plane wave propagation, $c=340 \frac{\mathrm{m}}{\mathrm{s}}$ is the speed of sound in air, $D=315 \mathrm{~mm}$ is the diameter of the duct and $\bar{U}$ is the mean air flow speed in the duct. Neglecting $U$ for this case will result in $f=632 \mathrm{~Hz}$. Thus in order to use single channel ANC for the ventilation duct the duct should only be excited with a broadband noise within the range [0-632] Hz. The lower limit of the noise frequency is dependent upon the capability of the loudspeakers to produce sound in the lower frequency range.

Linearity issues are also important to consider in order to maintain a high performance in most of the signal processing algorithms involved in ANC. For instance the aforementioned forward and feedback paths are assumed to have linear transfer characteristics. In reality the components such as loudspeakers and microphones may introduce nonlinearity in the transfer paths which cannot be ignored [15]. The actual properties of these transfer paths may be studied with the aid of frequency response function estimate and a coherence function estimate between the reference and error microphone signals. The frequency response function estimate helps in identifying the frequency region where the noise inhibit most of its power, i.e., this frequency region is where ANC will be most effective. The coherence function, on the other hand, is used to assess how much of the channel estimate can be described with linear terms. If the coherence is low for some frequencies, it implies that the estimated channel cannot be properly modeled using a linear model. Fig. 10, shows a typical coherence and frequency response function between reference and error microphone signals for a frequency range of [0- 200$] \mathrm{Hz}$. It is clear from the coherence function plot that, small coherence values below 50 $\mathrm{Hz}$ may indicate a nonlinear behavior of e.g., the loudspeaker or microphone or both at these frequencies. The frequency response function also suggest the modes or frequencies, for which ANC can be effectively used, lie around $80,120,160$, and $200 \mathrm{~Hz}$, as most of the noise power is concentrated at these frequencies [16].

\section{E. Implementation of ANC}

Once the aforementioned steps are performed implementing ANC is straight-forward. The required configuration for ANC according to Fig. 6, and the corresponding selection of equipment on the Measurement and Configuration Client as shown in Fig. 10, is performed. After the experimental setup is configured the RDE is launched similar to the forward path estimation. The developing of the code, Build, and Run etc. are also similar to the forward path estimation. Fig. 11, shows a single channel feed forward ANC results obtained using the filtered-x LMS algorithm during remote experiment on the ventilation duct. A $26 \mathrm{~dB}$ of attenuation obtained at $80 \mathrm{~Hz}$ can be clearly seen from the Fig. 11.

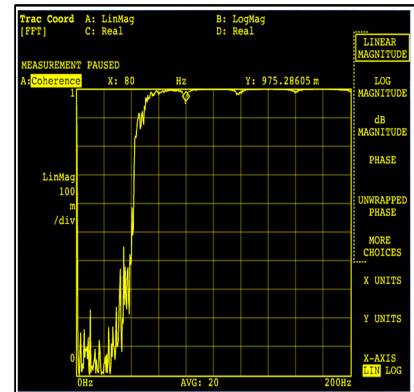

Coherence

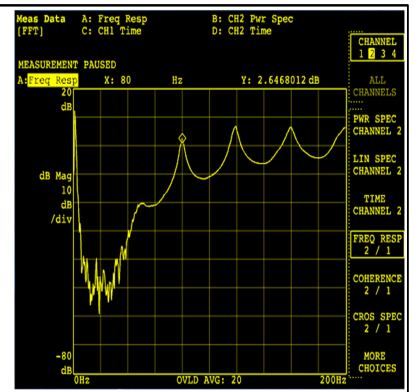

Frequency Response Function
Figure 10. Coherence and Frequency Response Function plots measured before forward path estimation. 


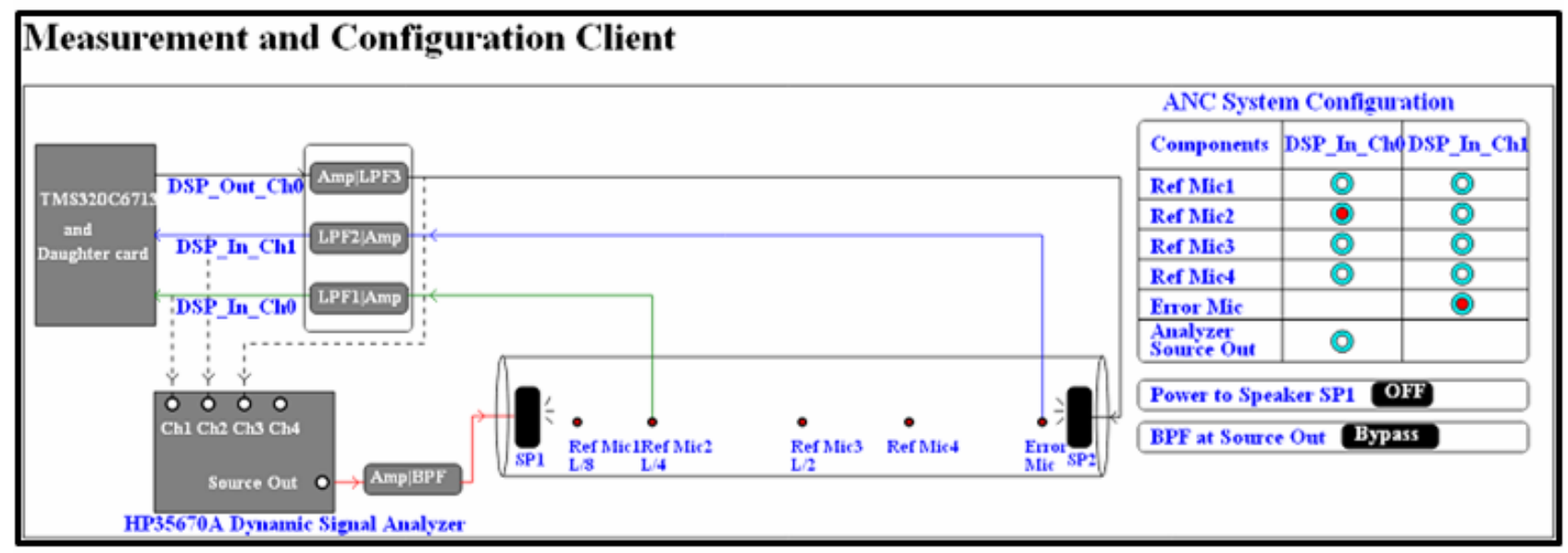

Figure 11. Screen shot of the Measurement and Configuration Client showing selection of the loudspeaker and microphones while performing ANC

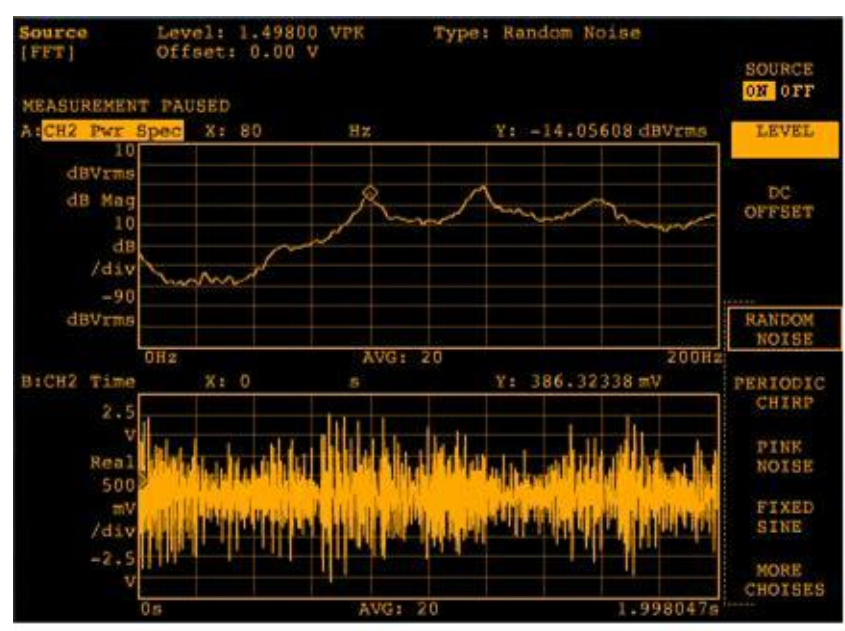

Before ANC

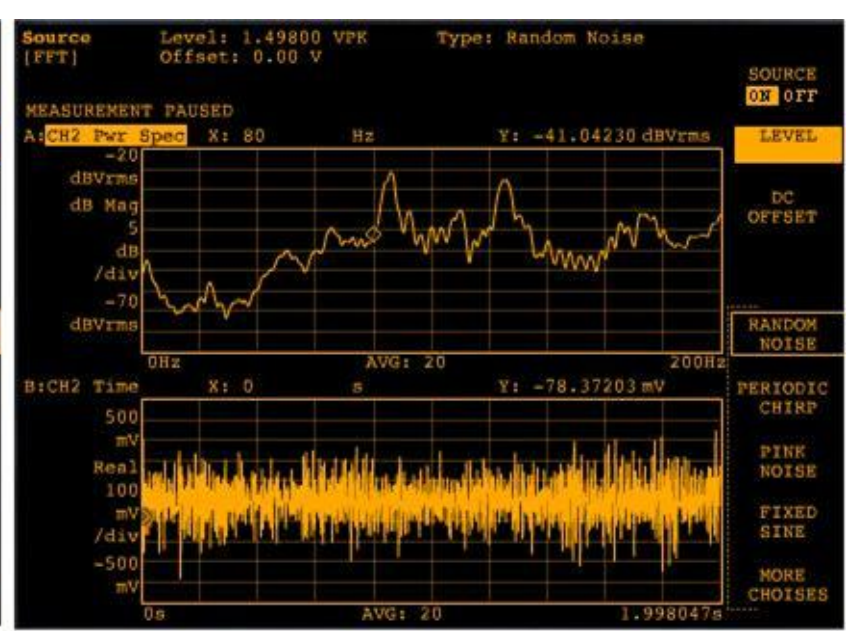

After ANC

Figure 12. Screen shots of the Signal analyzer showing the error signal both in time and frequency domain before and after ANC for the ventilation duct

\section{ACOUSTIC EXPERIMENTS}

The ventilation duct is $4.05 \mathrm{~m}$ in length and $315 \mathrm{~mm}$ in diameter and open at both the ends. The microphones and speakers installed at different positions along the length of the duct provide the possibility of the ANC laboratory setup to be used for a wide range of acoustic experiments. Based on the dimensions of the duct the students can verify and understand plane wave propagation, mode shapes and standing waves produced in the duct. As an example, the student can calculate the acoustical modes of the duct as shown in Fig.13, in the axial direction usingthe relation

$$
f=\frac{n c}{2 L} .
$$

Where $f$ is the nth acoustical harmonic frequency, $c=340 \frac{\mathrm{m}}{\mathrm{s}}$ is the speed of sound in air, $n=1,2,3 \ldots$, is a positive integer representing the resonance node and $L$ is the length of the duct. The Eigen frequency or modes of the duct by using (5) are $42 \mathrm{~Hz}$, $84 \mathrm{~Hz}$ and $126 \mathrm{~Hz}$ respectively. These frequencies are in line with the modes according to the frequency response function in Fig. 10. Furthermore, these experiments help students to familiarize themselves with the different measurement quantities commonly used in acoustics such rms, deciBel and DBSPL etc. by utilizing the signal analyzer remotely.

The power spectral density (PSD) of noise in the duct can be estimated using the signal analyzer module integrated to the Measurement and Configuration Client. Students shall compare the PSD estimate from the hardware to a theoretical PSD estimate.

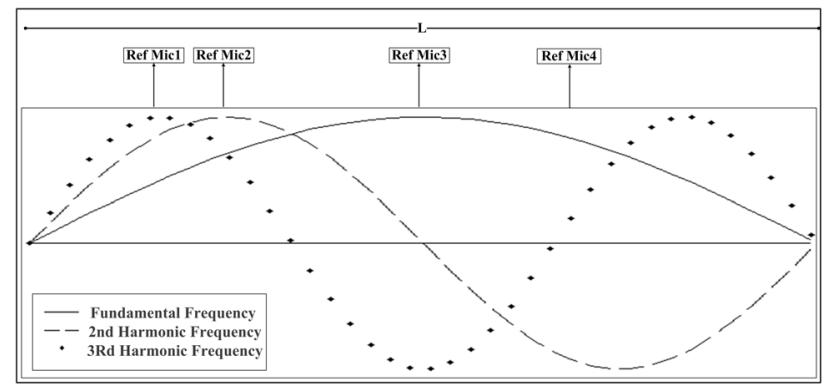

Figure 13. Acoustical mode shapes of along the length of the duct. 


\section{Digital Signal Processing ExPERIMENTS}

The proposed remote laboratory can be used to perform a range of digital signal processing experiments ranging from a beginner's simple data acquisition experiments to implementation of advanced adaptive signal processing techniques. The remote development environment is developed with an objective to program, debug and thereby control the digital signal processor i.e. TMS320C6713 in the laboratory from remote computer. The digital signal processor used in the remote laboratory is a general purpose floating point processor from Texas Instruments with fixed point processing modules (e.g., ALU, multiplier, etc.) on board as well. The proposed remote laboratory can thus also be used for students to train on DSP programming, optimization and memory management, in general.

The RDE is a graphical user interface to access the digital signal processor remotely. The remote debug environment exposes students to a realistic environment for implementing signal processing modules like FIR filter, IIR filter, as opposed to mathematical simulation models. Real time implementation of filters has challenges from several aspects, such as, the user has to consider the input signal

range of ADC, the sampling frequency for ADC and $\mathrm{DAC}$, the acquiring and buffering of data samples from the DSP's interrupt routines and the actual filter implementation. Further, advanced users can improve their optimization techniques and try to implement their optimized algorithms and evaluate against standard algorithms.

Apart from signal processing based experiments, the remote debug environment can also be used to develop embedded device drivers like general purpose drivers, general purpose timer modules, etc. Such experiments would help students study the processors inbuilt hardware

The proposed remote laboratory thus provide not only a bench for acoustic and ANC experiments, it is also an effective learning tool for DSP courses ranging from the beginners level to advanced level and research.

\section{CONCLUSION}

The proposed laboratory concept, built upon standard components and interfaces brings new knowledge and benefits to the community of remote laboratories. The remote laboratory presented in this paper enables students to enhance their learning and acquire experience on working with real systems and equipment in a wide range of fields. Students are exposed to the technical challenges involved in Active Noise Control, perform acoustic experiments and use a DSP platform remotely. The laboratory can be accessed through a standard web browser without involving extra costs for universities or students. The proposed system is based on the flexibility of the VISIR platform, developed by BTH and can be easily reconfigured and extended to suit more advanced and industrially oriented applications, such as, multichannel noise control, spatio-temporal acoustic experiments, multi-core DSP systems, and other types of sensors/actuators systems such as remote condition monitoring. One could also go the other way and shrink the system to suit a more compact setup, e.g., noise control in hearing defenders, which means the physical lab space required by such a system would shrink to the size of a desk. In a miniaturized setup, other system factors, such as, current consumption, number of DSP operations per second, optimization, and fixed point processing, would become of large interest, thus attracting another range of clients.

\section{REFERENCES}

[1] J. Ma and J. V. Nickerson, 'Hands-on, simulated, and remote laboratories: A comparative literature review’, ACM COMPUT. SURV, vol. 38, no. 3, p. 7, 2006. http://dx.doi.org/10.1145/ $\underline{1132960.1132961}$

[2] L. Gomes and S. Bogosyan, 'Current Trends in Remote Laboratories', Industrial Electronics, IEEE Transactions on, vol. 56, no. 12, pp. $4744-4756$, Dec. 2009.

[3] L.D. Feisel and A.J. Rosa, 'The role of the laboratory in undergraduate engineering education', Journal of Engineering Education, vol. 94, pp. 121-130, 2005.

[4] M. McAfee and S. Reid, 'Challenges in Providing Practical Labs Online to Distance Learning Students', presented at the 3rd International Symposium for Engineering Education, University College Cork, Ireland, 2010.

[5] A. Nafalski, Z. Nedic, J. Jan Machotka, Ö. Göl, A. Scarino, J. Crichton, I. Gustavsson, J. M. Ferreira, D. Lowe, and S. Murray, 'International Collaboration in Remote Engineering Laboratories: an Approach to Development', presented at the IEEE Transactions on Learning Technologies, 2009.

[6] A. Baccigalupi, C. De Capua, and A. Liccardo, 'Overview on Development of Remote Teaching Laboratories: from LabVIEW to Web Services', in Instrumentation and Measurement Technology Conference, 2006. IMTC 2006. Proceedings of the IEEE, 2006, pp. $992-997$.

[7] I. Gustavsson, J. Zackrisson, H. Åkesson, L. Håkansson, I. Claesson, and T. Lagö, 'Remote operation and control of traditional laboratory equipment', International Journal of Online Engineering, vol. 2, no. 1, p. 8 pp., 2009.

[8] Y. Yan, Y. Liang, X. Du, H. S. Hassane, and A. Ghorbani, 'Putting labs online with Web services', IT Professional, vol. 8, no. 2, pp. 27 -34, Apr. 2006. http://dx.doi.org/10.1109/ MITP.2006.45

[9] I. Gustavsson, T. Lagö, L. Håkansson, J. Zackrisson, and I. Claesson, 'The VISIR project - an Open Source Software Initiative for Distributed Online Laboratories’, in REV 2007, 2007.

[10] A. F. Almarshoud, 'The advancement in using remote laboratories in electrical engineering education: a review', European Journal of Engineering Education, vol. 36, no. 5, pp. 425-433, 2011. http://dx.doi.org/10.1080/03043797.2011.604125

[11] M. Tawfik, E. Sancristobal, S. Martin, C. Gil, A. Pesquera, P. Losada, G. Diaz, J. Peire, M. Castro, J. García Zubia, U. Hernández, P. Orduña, I. Angulo, M.A. Marques, M.C. Viegas, and G.R. Alves, 'VISIR: Experiences and Challenges', International journal of online engineering, vol. 8, no. 1, pp. 25-32, Feb. 2012.

[12] U. Hernandez-Jayo, J. García-Zubia, I. Angulo, D. Lopez-deIpiña, P. Orduña, J. Irurzun, and O. Dziabenko, 'LXI Technologies for Remote Labs: An Extension of the VISIR Project', International Journal of Online Engineering (iJOE), vol. 6, no. 5, pp. pp. 25-35, Aug. 2010.

[13] 'Telemanipulator for Remote Wiring of Electrical Circuits (Conference Paper by Ingvar Gustavsson, Johan Zackrisson, Josef Ström Bartunek, Kristian Nilsson, Lars Håkansson, Ingvar Claesson, Thomas L Lagö) - Electronic Research Archive@ Blekinge Institute of Technology (BTH)'. [Online]. Available: http://www.bth.se/fou/forskinfo.nsf/all/48e19e62e3b88fd7c1257 4a5003270e0?OpenDocument. [Accessed: 05-Oct-2012].

[14] 'NI LabVIEW DSP Test Integration Toolkit', National Instruments. [Online]. Available: http://www.ni.com/whitepaper/5839/en. [Accessed: 05-Oct-2012].

[15] S. J. Elliott and P. A. Nelson, 'Active noise control', IEEE Signal Processing Magazine, vol. 10, no. 4, pp. 12 -35, Oct. 1993. http://dx.doi.org/10.1109/79.248551

[16] C. H. Hansen and S. D. Snyder, Active control of noise and vibration. London: Spon, 1997. 
[17] J. Landaluze, I. Portilla, J. M. Pagalday, A. Martı\&\#x0301;nez, and R. Reyero, 'Application of active noise control to an elevator cabin', Control Engineering Practice, vol. 11, no. 12, pp. 1423-1431, Dec. 2003. http://dx.doi.org/10.1016/S0967-0661 (03)00077-7

[18] M. H. Hayes, Statistical digital signal processing and modeling. New York: Wiley, 1996.

[19] Sen M. Kuo and Dennis R. Morgan, Active noise control systems: algorithms and DSP implementations. Wiley, 1996.

[20] H. P. Wallin, U. Carlsson, M. Åbom, H. Boden, and R. Glav, Sound and Vibration, Second. .

\section{AuTHORS}

I. Khan is with Blekinge Institute of Technology, Karlskrona, Sweden (imran.khan@ bth.se).

D. Muthusamy is with Blekinge Institute of Technology, Karlskrona, Sweden (dsp.dineshkumar@gmail.com).
W. Ahmad is with Blekinge Institute of Technology, Karlskrona, Sweden (engr.waqas86@gmail.com).

B. Sällberg is with Blekinge Institute of Technology, Karlskrona, Sweden (benny.sallberg@bth.se).

K. Nilsson is with Blekinge Institute of Technology, Karlskrona, Sweden (kristian.nilsson@ bth.se).

J. Zackrisson is with Blekinge Institute of Technology, Karlskrona, Sweden (johan.zackrisson@ bth.se).

I. Gustavsson is with Blekinge Institute of Technology, Karlskrona, Sweden (ingvar.gustavsson@bth.se).

L. Håkansson is with Blekinge Institute of Technology, Karlskrona, Sweden (lars.hakansson@bth.se).

This article is an extended and modified version of a paper presented at the International Conference on Remote Engineering \& Virtual Instrumentation (REV2012), held at University of Deusto, Bilbao, Spain, July 4-6, 2012. Manuscript received 08 October 2012. Published as resubmitted by the authors 28 November 2012. 